\title{
Empirical research on human behavior changes and digital intervention by one-way car-sharing
}

\author{
Yutaka Arakawa *
}

\begin{abstract}
In this paper, we summarize our ongoing project called IS^3 (Incentive-based Intelligent Intervention for Smart and Sustainable Societies), which was started in February 2016. This project is empirical research that aims to utilize human behavior changes for solving social problems and sustainably maintaining society. As a sample society, we prepared a one-way car-sharing system between our campus and the neighboring train station. Our society's main problem is the cost of ferrying cars to balance the number of vehicles parked in each parking lot. To solve this problem, we encourage potential users to move cars to address imbalances. In this paper, we explain the overview of this project and its challenging points.

Keywords: Behavior change, One-way car-sharing, Activity recognition, Incentive mechanism, Gamification
\end{abstract}

\section{Introduction}

The advent of ubiquitous societies continues to draw nearer. Reflecting the rapid progress of information technologies, many sensors are being deployed in modern living areas. We are already wearing such sensors as smartphones and smart watches. Many types of research have addressed activity recognition $[8,10]$. Various context-aware services are improving the convenience of our lives by estimating our physical and mental state. On the other hand, modern societies are also suffering from problems caused by low birthrates and aging populations. Due to shrinking tax revenues, in many places, maintaining services is a critical problem. For example, the amount of obesity and garbage are increasing yearby-year, and the cost of maintaining public transportation is burdening local government finances. We believe that behavior changes, which are possible solutions, can be intentionally encouraged by Artificial Intelligence(AI).

Our behaviors have already been affected by AI. We determine driving routes based on the line colors (red denotes congestion) on maps because we trust the information from cyber systems. We never check the actual traffic. A company or AI could control a city's traffic by just changing the line colors on the map. Pokémon GO is another example that shows that our behavior has already been affected by AI. To capture monsters in a cyber-world, people must literally walk around in their physical worlds. Microsoft research reported that

* Nara Institute of Science and Technology, Nara, Japan 


\section{Empirical research in a small actual society}

Confirming the ability of Incentive-based intentional Intervention for solving social problems.

\section{Digital Intervention}

Intentionally intervene a human behavior using activity recognition and incentives.
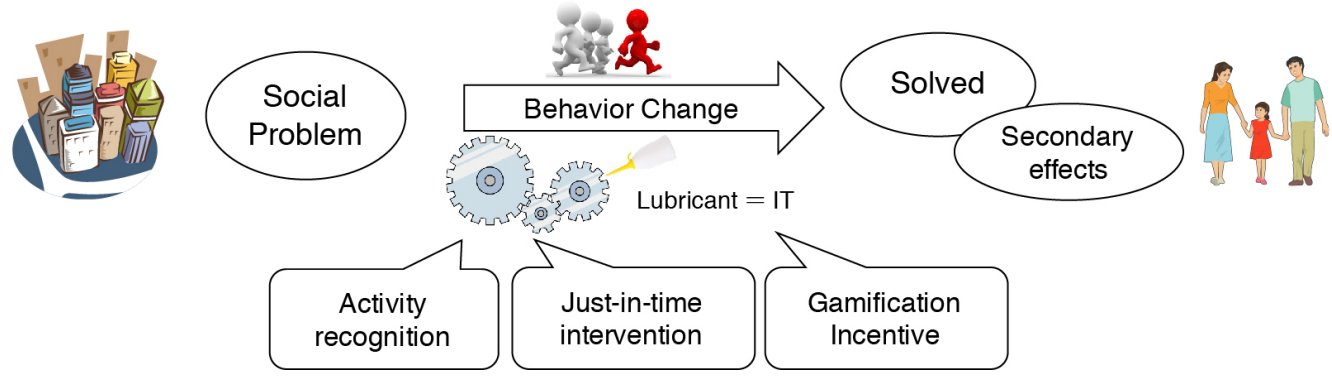

Figure 1: Project overview: human behavior changes for maintaining society

the game has encouraged people to do more walking [2]. The Apple watch has a function called "stand" that suggests that its owners stand up for their health when they have been sitting for more than one hour. Even though it might seem slightly incongruous for a watch to give orders to its owner, we are likely to comply if a clear benefit exists. Such wearables are called "digital medicine [7]" that has the potential to modify our living habits to reduce lifestyle-related diseases. Since the increase of medical and social welfare costs ishuge social problems, digital medicine is attracting attention.

Based on these research backgrounds, we propose a novel approach that actively intervenes in human behavior by utilizing various information technologies and small incentives for solving social problems (Fig. 1). To realize this concept, research is still required in the areas of human activity recognition, just-in-time intervention mechanisms, and gamification. In addition, since conducting experiments and evaluations is usually difficult because our target is an actual society. We prepared an experimental society on which our related research can be conducted. Our project's goal is to empirically confirm the potential of human behavior changes by AI and determine the ability of digital intervention for sustainably maintaining our society. In the rest of this paper, we describe related work in the second section. We explain our experimental society in the third section. Next, we discuss the expected and estimated research topics in the fourth section and summarize our research project in the final section.

\section{Related work}

In this section, we introduce some related work for each technical challenge.

\subsection{Activity recognition}

The first technical challenge is activity recognition on which research already exists in smart home environments $[14,10,8]$ where various sensors are embedded. By combining power consumption sensors, indoor position measurement sensors, and open/close sensors, about 10 to 20 kinds of human activities are being estimated with about $90 \%$ accuracy.

However, our target activity is rough movement patterns in daily life, not the detailed activities treated in the above research. To identify potential users who might be willing to ferry cars, we need to predict future human behaviors based on past behaviors. Various 
methods have already been proposed $[5,12,17]$. UberPOOL ${ }^{1}$ has already started to use human behavior predictions for promoting affordable ride sharing [9], in which the destination of potential users is predicted by machine learning.

On our project's mobile application for reservations will also collect the trajectory patterns of daily movements. We will also extract the movement patterns of each user for deciding the best person to ask to ferry a car.

\subsection{Investigation of interruptability}

The second technical challenge is how to interrupt a person by presenting information to him. Currently, since we receive too many notifications on our smartphones. We often ignore them. "Interruptability" denotes the possibility to interrupt the current human behaviors. To improve interruptability, a system has to recognize human activities and send information at proper timing [13].

We conducted a survey on context and interruptability for one week in 2015 [16], and regularly sent messages to ten participants and measured their response times without informing them that they were part of an investigation. After a week, we explained the survey's purpose, and distributed questionnaires about their contexts at the reply times. We confirmed that interruptability depends on the context. For example, if a student is with her family, it is difficult to interrupt her by a message.

In our project, we have to consider different levels of interruptability among individuals because a user's motivation, interest, and ambition as well as his context (location, time) will affect his decision to accept/reject the request.

\subsection{Gamification and incentive mechanism}

Gamification is an effective approach for attracting people with lower incentives to various activities. For example, we installed a gamification mechanism in several participatory sensing applications [15,3]. Through empirical experiment, we confirmed that gamification generated competitive energy and successfully suppressed monetary incentives. Our other research adopted gamification into "behavior change"[11], for example, a diet method based on food journaling. However, recording everything one eats is troublesome. Therefore, we proposed using an animal avatar that grows up if a user records his daily food intake: the avatar becomes inactive if daily updates are neglected.

In our project, we introduce a gamification mechanism that efficiently prompt human behavior changes. For example, points required for reserving a car can be earned by cooperating with the requests of others' request.

\subsection{One-way car-sharing}

Car-sharing is attracting much attention for its rapid growth potential in future sharedeconomies. Zipcar ${ }^{2}$ and Car$_{2} \mathrm{Go}^{3}$ are pioneers in this field. In Japan, Times Car Plus ${ }^{4}$ has already deployed 15,000 shareable cars. These car-sharing services can be classified into two categories round-trip and one-way. As implied by the name, one-way allows a user to just go to a different station at which he rented the car. However, most are round-trips since

\footnotetext{
${ }^{1}$ https://www.uber.com/en-JP/ride/uberpool/

${ }^{2}$ http://www .zipcar.com

${ }^{3}$ https://www. car2go.com/

${ }^{4}$ https://plus.timescar.jp
} 
balancing the number of cars and parking spaces is difficult. If all the cars at the parking space A are moved and parked at other spaces, the service provider must supply a new car for the parking space A. If the parking space A becomes full, the service provider must move a car to make space for a potential user who wants to get off at parking space A. These tasks are called "car ferrying", and are costly for service providers.

In our project, we target a one-way car-sharing system and solve the car ferry problem by changing human behavior.

\section{One-way car-sharing system as empirical society}

Figure 2 introduces our experiment with a social system and its customized car-sharing system that is composed of three electric vehicles and two parking spaces. We assume a small society where the university personnel and students are living as its citizens. A special smartphone application provides a reservation function and also works as a key. All reservations are managed by Contribution Points (CPs).

Citizens of this society can enjoy its services, i.e., a car, with CPs. Our university's campus is located on a mountainside and has few buses. Therefore, a car-sharing service is quite attractive. This society has several problems, such as unbalanced supply and demand. Citizens can earn CPs by cooperating to solve problems.

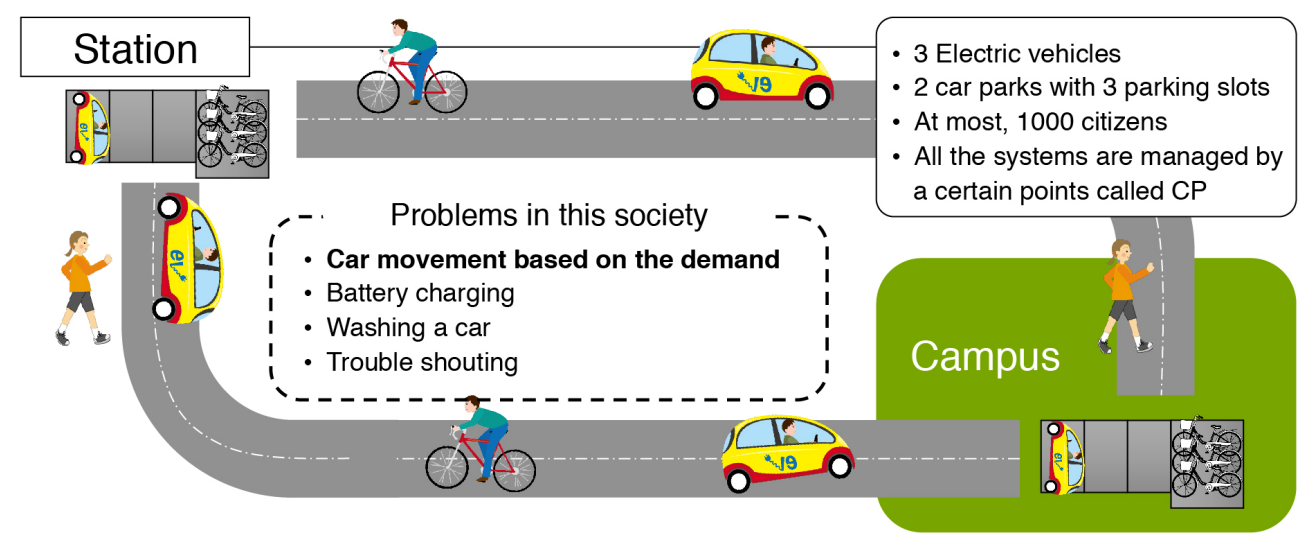

Figure 2: One-way car-sharing system introduced in our university

\subsection{System architecture}

\subsubsection{Electric vehicles}

In this society, electric vehicles denote both services and rewards. Therefore, we have two different types of cars (Fig. 3): a Mitsubishi i-MiEV and a BMW i-3. The former is used as both a basic service and a basic reward, and the latter denotes a higher reward for increasing a user's motivation to contribute to society.

If a citizen's contribution to society is large, she can obtain a special reward: a ticket for driving a BMW i-3. All of the cars are equipped with a 3G-connected OBDII (on-board diagnostics) sensor for monitoring location and status in real-time management software. A dual camera drive recorder always simultaneously records both outside and inside. A car key is customized for unlocking the vehicle from the smartphone application. 


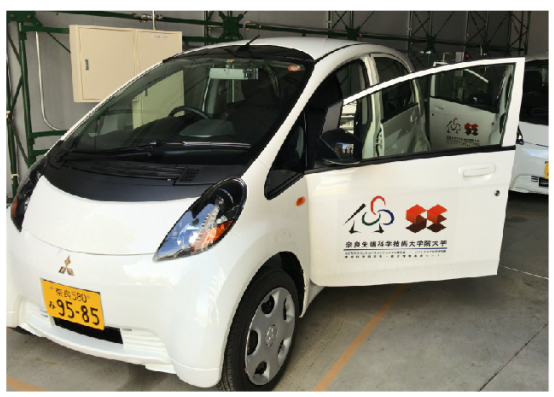

Mitsubishi i-MiEV $\times 2$

- Basic service (up to $80 \mathrm{~km}$ distance)

- Basic reward

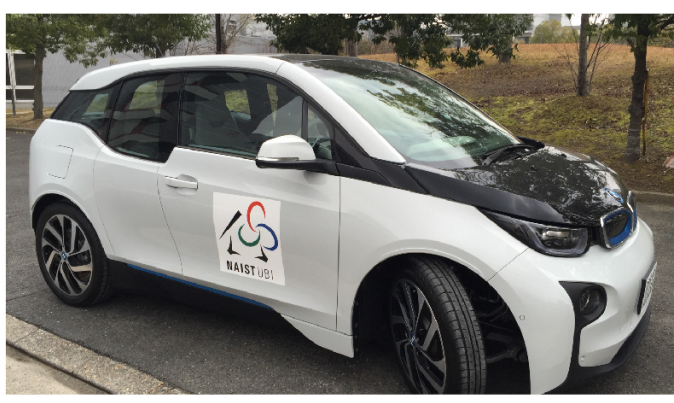

BMW i-3 $\times 1$

- Special service (up to $150 \mathrm{~km}$ distance)

- Special reward (high power and luxury)

Figure 3: Cars and rewards in this society

\subsubsection{Car parking lots and battery chargers with automatic authentication}

To simplify our society's problem, we built two parking lots with ample parking slots: inside the campus, and near the train station (Fig. 2). All of the parking lots are equipped a standard battery charger. The battery chargers and the cars are customized for automatic authentication called "Ubiden," where a car transmits a certain ID signal on such electric cable called Power Line Communication (PLC), and a battery charger only provides power for eligible cars after checking the received ID by a $3 \mathrm{G}$ connection.

\subsubsection{Types of trips}

Our system provides two types of trips. One is "a user-reserved trip" which is requested by the user self. When the reservation is made, a user spends CPs that he has already earned. Another is "a system-requested trip" issued by the system for solving a problem of the society. If the user who received the request agrees to it, he can use a car without any CPs or might earn CPs.

However, since we assume that satisfying a system-request is difficult because the system must intentionally change human behavior, we assume two types of system-request trips.

An easier approach is a change request for a user-reserved trip. The system asks a user who has already reserved a car for a different time slot to change his reservation time. If his purpose is just shopping, he might accept the system's request.

A more difficult approach is a request to a user who doesn't have a reservation. In this approach, a request suddenly arrives by a user's smartphone who has no plans to use a car based on behavior pattern prediction. We assume the acceptance rate of such requests will be low. However, some users might accept if the timing is good because the requested user is already a member of our system and has a potential to use a car.

\subsubsection{Contribution points}

Contribution points (CPs) are the virtual currency in this society. Citizens who want to use a car earn points by contributing to society such as car ferrying and car washing. Also, the number of CPs required for renting is based on the type of car. A basic car (i-MiEV) requires just a few $\mathrm{CPs}$, but a special car (BMW i-3) requires more CPs. The $\mathrm{CP}$ design is 
a critical part of this project because it fuels behavior change. We have a rough design of CPs, which is explained in the next section, but it isn't operational yet. We are currently distributing CPs manually to each user by administrator account.

\subsubsection{Behavior monitoring and prediction}

The mobile application for reserving a car works as a key and a behavior tracker and periodically uploads the user's location to the cloud (Fig. 4). The system analyzes the uploaded data and learns the time each user reaches the university, and returns home, her usual mode of transportation, and the nearest train station. The system also analyzes the weather, the day of the week, and the past acceptance rates against system-requested trips. Based on such analysis, the system periodically determines the best person to request to ferry a car.

We expect to identify the behavior patterns of the target users (university personnel and students) because they are moving between their home and the campus based on their respective schedules.

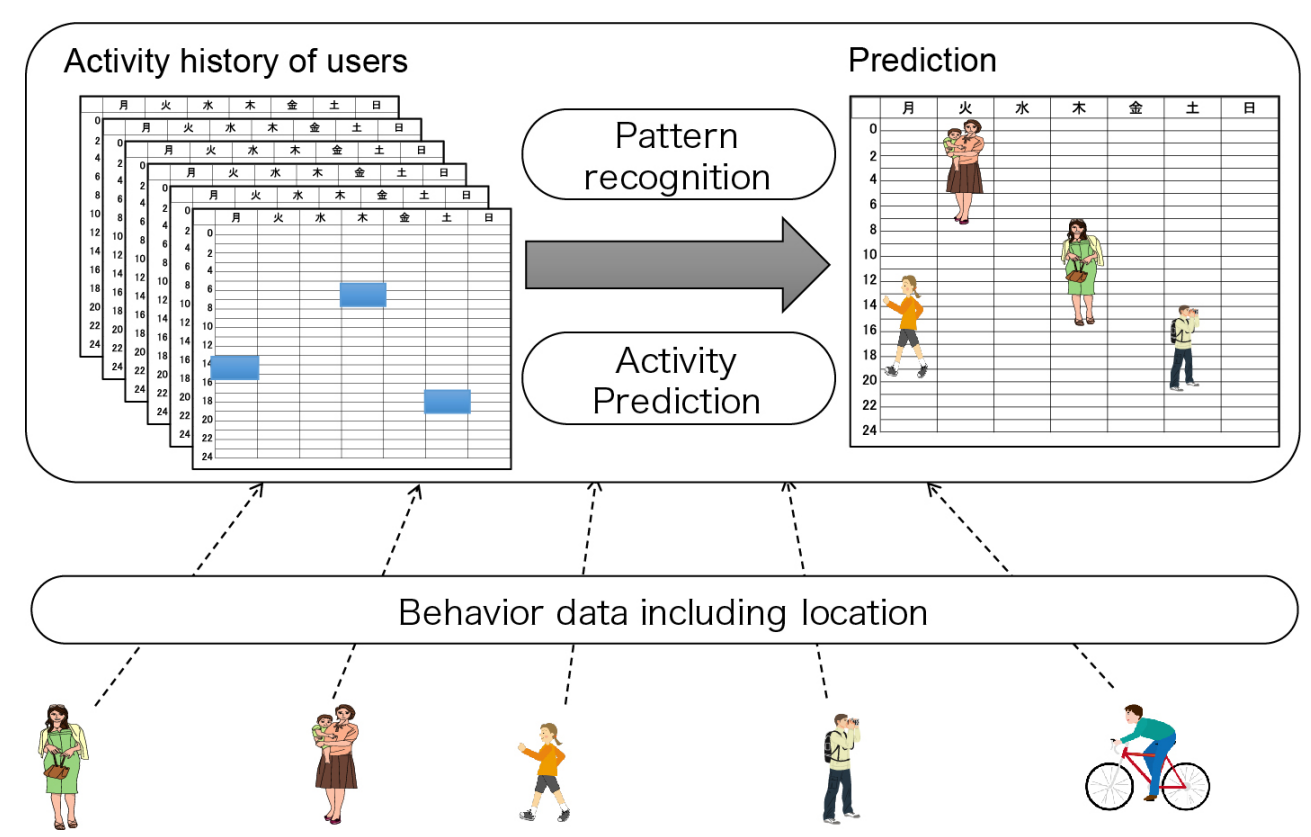

Figure 4: Behavior pattern analysis on the cloud

\subsection{System flow}

In our society, all the procedures for using a car are conducted through the mobile application. For the reservations, we use the virtual points called CPs instead of actual currency.

Figure 5 shows the procedure for obtaining a car with the mobile application. Three buttons appear on its top menu. In the reservation windows, a user selects a car (1) and a desired time (2). At this moment, the points required for reserving a car are calculated. The points that are required for a unit time can be manually changed by an administrator. In the future, we will introduce dynamic pricing, which is automatically based on demand. 


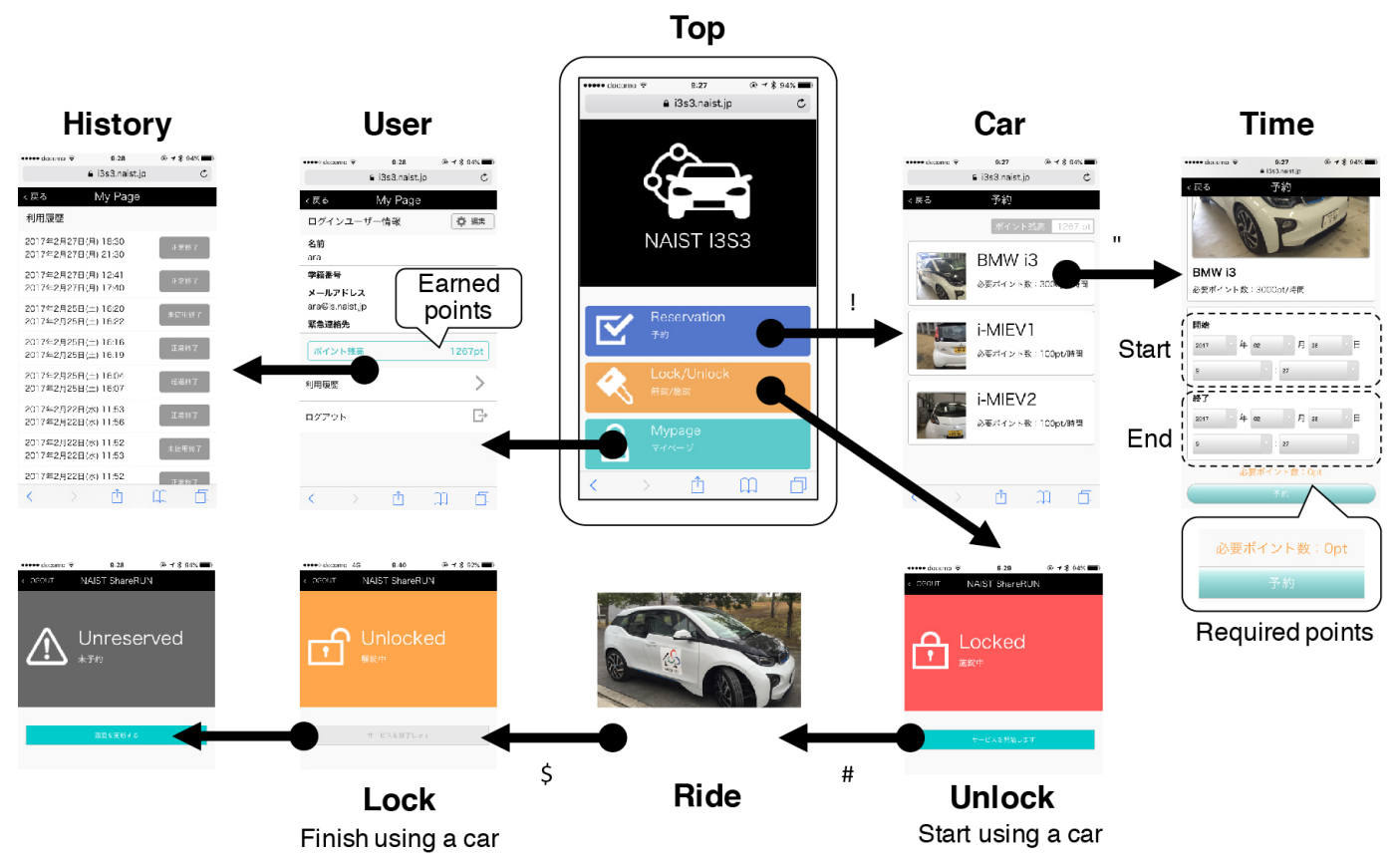

Figure 5: Mobile application for this society and usage flow

The mobile application not only provides a reservation function but also acts as a key. After reserving a car, the user goes to the lock/unlock function. If the reservation is valid, the button appears that unlock the reserved car. The communication between the mobile application and the cars is a bluetooth low energy(BLE). If the authentication is successful (3), the car is unlocked, and the key is disabled. After the user finishes using the car, she uses the mobile application again to lock it (4)).

On the "my page" function of the application, users can check and modify such information as name and e-mail address and confirm earned CPs and rent history.

\subsection{Digital intervention for behavior change}

Figure 6 shows an expected scenario in our society. The above mobile application for reservations is equipped with a behavior recognition engine, and daily behavior patterns are continuously collected by our cloud. Since the location of cars is shared in real-time, AI can easily detect problems in this society, i.e., an unbalance of cars at the two parking lots. In this figure, the problem is that since all three cars are in the campus's parking lot, no one can drive from the station.

To solve this problem, AI first finds a potential user with the highest probability of getting on the road at that time by a data analysis of past behavior patterns. Actually, the behaviors of the students and university personnel might be patterned based on the day of the week and the weather.

After deciding the target user, AI sends a message and asks her to take a car from our campus to the neighbor station. This is called a digital intervention. If she agrees to drive the car to the station, the social problem is solved by a single message. 


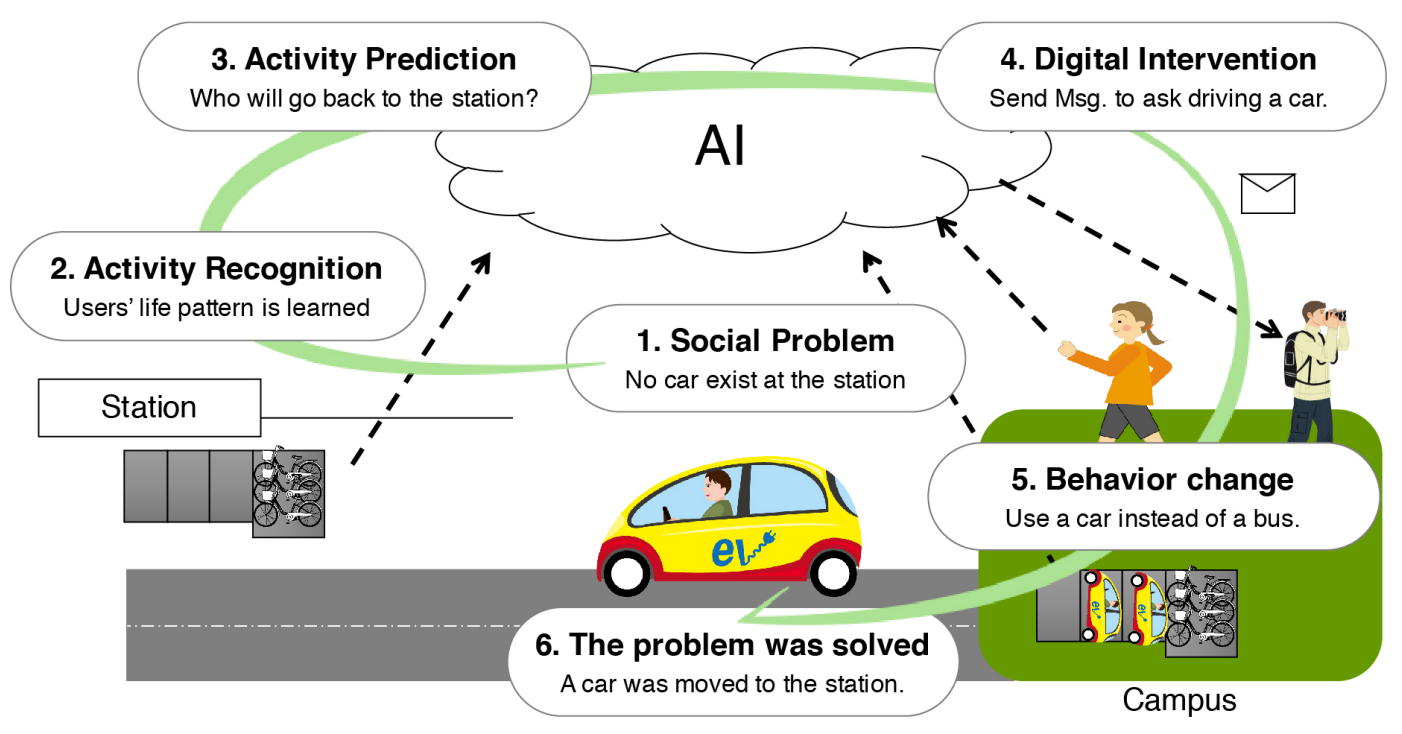

Figure 6: Assumed scenario of digital intervention for behavior changes

\section{Future research topics}

The expected duration of this project is four years, and we and some university colleagues are planning to tackle the following research topics. First, we will empirically confirm the ability of digital intervention to change human behaviors. Second, we will try various strategies to maximize the working ratios of the service by minimizing the maintenance cost.

\subsection{Recognition and prediction of human behavior pattern}

We will first develop a system for recognizing and predicting human behaviors by extending the mobile application to sense the trajectory patterns of each user like Moves ${ }^{5}$. To protect privacy, we will only collect data related to our society, including the times each user comes to the university and returns home, the normally used stations, the usual mode of transportation, and the past usage history with our car-sharing service.

From the collected data, we will extract behavior patterns by applying recurrent neural networks(RNNs), such as long short-term memory(LSTM). LSTM was already used for solving a similar problem $[6,1]$. Finally, we will identify potential users who might drive a car based on such varying conditions as location, time, and weather.

\subsection{Just-in-time digital intervention}

To efficiently change behaviors, a valid request should be sent to an appropriate person at proper timing. In our previous work [16], we confirmed that proper timing for deciding participation depends on the user's context. In this project, we will measure the interruptability of each user at various conditions (location and timing). In addition, we need to consider the acceptability of reward at various conditions because a person might accept a request with a small reward, but others may decline the same request even with a higher reward.

\footnotetext{
${ }^{5}$ https://moves-app.com/
} 


\subsection{Design of CP-based society}

Since our society is based on CPs, we need to design models for acquiring and consuming them. A large amount of CPs might prompt users to cooperate easily for solving problems. However, the capacity of service is limited because our society only has three cars that require eight hours of charging time. Therefore, a large amount of CPs could fuel a point inflation and reduce motivation.

\subsubsection{Earning model of $C P$}

Users earn CPs by contributing to society. At this moment, only ferrying a car is considered as a contribution. However, in the future, we will include such other actual opportunities/problems as car washing and car maintenance as problems of society and give CPs if users cooperate to solve them.

In the future, we will also introduce various behaviors that can earn CPs. For example, walking is a good health care practice. Therefore, we might give CPs if a user walks a number of steps that exceed a particular threshold. The number of commitments on Github could also be a parameter for giving CPs, because they are contributions to the software community. However, we need to consider the balance of CPs for different behaviors. A user might choose behaviors that are easy and give many CPs.

\subsubsection{Consuming model of CPs}

CPs are spent by using a car. The CPs required for renting a high performance and ordinary cars are different. In addition, CPs are consumed by such breaches as exceeding the return time and forgetting to recharge a battery.

We haven't yet concretely defined the $\mathrm{CP}$ values for each contribution and consumption. We will conduct various experiments that change the values for the behaviors and breaches to heuristically find adequate parameters.

\subsubsection{Gamification for reducing the required CPs}

Based on our previous work [15] and survey [4], we will design a gamification scenario that reduce the amount of required CPs. For example, a user who continuously contributes to society can obtain more CPs for identical behaviors. To encourage continuous contributions, both levels and rankings will be introduced, like in social games.

\subsubsection{Dynamic CPs}

In addition, both obtainable and required CPs will be changed in conjunction with demand. If many users want to contribute to society, the amount of CPs will be small that can be obtained for such behavior. On the contrary, more CPs will be set to get contributions for more difficult requests like ferrying a car at night. The amount of required CPs will increase when many users want to use a car and decrease when nobody wants to use one. In the future, we believe that the above dynamic situation of CPs will be automatically optimized by AI. 


\subsection{Extended CPs for secondary effects}

A secondary effect is an additional and unconscious outcome that occurs in addition to the desired main effect. For example, our society asks a user to drive a car for ferrying it. If the user accepts the society's request, the desired main effect has been achieved. Simultaneously, this request causes such secondary effects as a walking to a parking lot and an opportunity to return home earlier than usual. If we introduce share-riding points as an extended reward, they might create new communication between students who are strangers.

We defince the following as secondary effects.

- Health : Extended CPs for health-related behavior

- Communication: Extended CPs to invite others for ride sharing.

- Research : Extended CPs for cooperating with research.

- Volunteers : Extended CPs for open source software communities.

\section{Evaluation Criteria}

In this section, we discuss the criteria for evaluating the pros and cons of our proposed system.

Our idea encourages behavior changes by information technology and their adoption for a part of the operation of social systems. In our experimental environment, we prepared a one-way car-sharing system and treated it as a society.

We identified two evaluation axes. The first axis, which evaluates how many behavior changes occurred by intentional information intervention based on activity recognition and prediction techniques, can be evaluated by comparing the acceptance rate with requests from the system. If activity recognition and prediction accuracy are low, accurate interventions are impossible. Since the acceptance rate will change according to people, feeling and weather, we believe that long-term observations are necessary.

Another evaluation is the utilization efficiency of a one-way car-sharing system. We must evaluate its impact on society by incorporating intentional behavioral changes. In carsharing systems, the objective function is maximizing car utilization under limited CPs. If a system uses unlimited CPs, CP inflation will inevitably occur. If so, the motivation of users to contribute to society and earn CPs will be dampened. Therefore, perhaps, our system can be evaluated as successful if we create a situation with good car utilization by maintaining a point balance.

\section{Conclusion}

We introduced a novel project at our university called IS^3, which determines the effect of information technologies for prompting changes in human behavior. Our ultimate goal is to introduce these techniques into our actual life for sustainably solving various social problems. We confirmed that various information technologies are required for intentionally encouraging behavior changes. Since we just started this project in February 2016, we have no achievements to report yet. However, we expect to obtain and report some typical strategies for utilizing human behavior changes for our future society. 


\section{Acknowledgement}

This work was partially supported by JST PRESTO, JSPS KAKENHI (15H05708). The following researchers also supported this project: Keiichi Yasumoto, Kenichi Matsumoto, Hideaki Hata, Manato Fujimoto, Hirohiko Suwa, and Kotone Senju. We acknowledge the stimulated discussion in the meeting of the Cooperative Research Project of the Research Institute of Electrical Communication, Tohoku University.

\section{References}

[1] Alexandre Alahi, Kratarth Goel, Vignesh Ramanathan, Alexandre Robicquet, Li FeiFei, and Silvio Savarese. Social lstm: Human trajectory prediction in crowded spaces. In Proceedings of the IEEE Conference on Computer Vision and Pattern Recognition, pp. 961-971, 2016.

[2] Tim Althoff, Ryen W White, and Eric Horvitz. Influence of pokémon go on physical activity: Study and implications. Journal of Medical Internet Research, Vol. 18, No. 12, 2016.

[3] Yutaka Arakawa and Yuki Matsuda. Gamification mechanism for enhancing a participatory urban sensing: Survey and practical results. Journal of Information Processing, Vol. 24, No. 1, pp. 31-38, 2016.

[4] Yutaka Arakawa and Yuki Matsuda. Gamification mechanism for enhancing a participatory urban sensing: Survey and practical results. JIP, Vol. 24, No. 1, pp. 31-38, 2016.

[5] Daniel Ashbrook and Thad Starner. Using gps to learn significant locations and predict movement across multiple users. Personal and Ubiquitous computing, Vol. 7, No. 5, pp. 275-286, 2003.

[6] Alexandre de Brébisson, Étienne Simon, Alex Auvolat, Pascal Vincent, and Yoshua Bengio. Artificial neural networks applied to taxi destination prediction. arXiv preprint arXiv:1508.00021, 2015.

[7] Eric Elenko, Lindsay Underwood, and Daphne Zohar. Defining digital medicine. Nature biotechnology, Vol. 33, No. 5, p. 456, 2015.

[8] Nakagawa Eri, Moriya Kazuki, Suwa Hirohiko, Fujimoto Manato, Arakawa Yutaka, and Yasumoto Keiichi. Toward real-time in-home activity recognition using indoor positioning sensor and power meters. In The First International Workshop on Pervasive Smart Living Spaces (PerLS 2017). IEEE, 2017.

[9] Travis Kalanick. Uber's plan to get more people into fewer cars (ted talk). https://www.ted.com/talks/travis_kalanick_uber_s_plan_to_ get_more_people_into_fewer_cars.

[10] Moriya Kazuki, Nakagawa Eri, Fujimoto Manato, Suwa Hirohiko, Arakawa Yutaka, Kimura Aki, Miki Satoko, and Yasumoto Keiichi. Daily living activity recognition with echonet lite appliances and motion sensors. In First International Workshop on Mobile and Pervasive Internet of Things (PerIoT 2017). IEEE, 2017. 
[11] Edith Talina Luhanga, Akpa Akpro Elder Hippocrate, Hirohiko Suwa, Yutaka Arakawa, and Keiichi Yasumoto. Happyinu: exploring how to use games and extrinsic rewards for consistent food tracking behavior. In Mobile Computing and Ubiquitous Networking (ICMU), 2016 Ninth International Conference on, pp. 1-7. IEEE, 2016.

[12] Emiliano Miluzzo, Nicholas D Lane, Kristóf Fodor, Ronald Peterson, Hong Lu, Mirco Musolesi, Shane B Eisenman, Xiao Zheng, and Andrew T Campbell. Sensing meets mobile social networks: the design, implementation and evaluation of the cenceme application. In Proceedings of the 6th ACM conference on Embedded network sensor systems, pp. 337-350. ACM, 2008.

[13] Tadashi Okoshi, Jin Nakazawa, and Hideyuki Tokuda. Interruptibility research: opportunities for future flourishment. In Proceedings of the 2016 ACM International Joint Conference on Pervasive and Ubiquitous Computing: Adjunct, pp. 1524-1529. ACM, 2016.

[14] Kenki Ueda, Hirohiko Suwa, Yutaka Arakawa, and Keiichi Yasumoto. Exploring accuracy-cost tradeoff in in-home living activity recognition based on power consumptions and user positions. In IEEE IUCC2015, pp. 1130-1137. IEEE, 2015.

[15] Yuki Ueyama, Morihiko Tamai, Yasuhiko Arakawa, and Kiyotoshi Yasumoto. Gamification-based incentive mechanism for participatory sensing. In First International Workshop on Crowdsensing Methods, Techniques, and Applications (CROWDSENSING) in conjunction with IEEE PerCom 2014, pp. 98-103. IEEE, 2014.

[16] Akaike Yuma, Arakawa Yutaka, Suwa Hirohiko, and Yasumoto Keiichi. Estimation of the response based on the context sensing. IPSJ Journal, Vol. 57, No. 2, pp. 543-552, feb 2016 .

[17] Yu Zheng, Quannan Li, Yukun Chen, Xing Xie, and Wei-Ying Ma. Understanding mobility based on gps data. In Proceedings of the 10th international conference on Ubiquitous computing, pp. 312-321. ACM, 2008. 